Valero, G., Patiño, R., y Vargas, H. (2020), Feminilización y Feminización de la Profesión Contable en Colombia. Contaduría Universidad de Antioquia, 76, 13-33.

Doi:https://doi.org/10.17533/udea.rc.n76a01

\title{
Feminilización y Feminización de la Profesión Contable en Colombia*
}

Gloria Milena Valero Zapata

gloriavalero@usantotomas.edu.co

Universidad Santo Tomás

orcid: 0000-0002-6796-3907

Ruth Alejandra Patiño Jacinto

rapatinoj@unal.edu.co

Universidad Nacional de Colombia

orcid: 0000-0001-6017-7666

Hasbleidy Vargas Toledo

hasbleidyvargas@usantotomas.edu.co

Universidad Santo Tomas

orcid: 0000-0002-0112-7957

* Este artículo de investigación es producto del proyecto titulado "Incidencia de los International Education Standards en la Feminilización y Feminización de la Profesión Contable en la Ciudad de Bogotá"; el cual fue financiado a partir de la convocatoria general para el fomento, desarrollo y producción de la investigación de alto nivel en la Universidad Santo Tomás, sede Bogotá, en el año 2018. 
Feminilización y Feminización de la Profesión Contable en Colombia

Resumen: El presente documento tiene como propósito analizar la feminilización y la feminización de la profesión contable en Colombia entre el 2001 y el 20181; entendiendo como feminilización el incremento en la participación de las mujeres en una determinada profesión y por feminización los cambios de significado asociados al aumento cuantitativo de la participación femenina. La investigación es cualitativa; en primera instancia tiene una perspectiva cualitativa que pretende comprender y explicar los procesos que han incidido en la feminización de la profesión contable en Colombia, a través del análisis documental; posteriormente es cuantitativa porque procura analizar las mediciones realizadas sobre dicho fenómeno. Frente a los datos analizados se encontró que las mujeres constituyen una mayoría en el campo contable, aspecto que no necesariamente se ve contrastado con la remuneración laboral.

Palabras claves: Feminilización de la profesión contable, Feminización de la profesión contable, Profesión Contable en Colombia, género, mujer contadora.

\section{Feminilization and Feminization of the Accounting profession in Colombia}

Abstract: The present document aims at analyzing feminilization and feminization of the accounting profession in Colombia between 2001 and 2018; understanding feminilization as the increase in women's participation in a given profession, and feminization as changes in meaning associated to the quantitative increase of women's participation. Firstly, the research has a qualitative perspective that seeks to understand and explain the processes that have influenced feminization of the accounting profession in Colombia, through a documentary analysis. Secondly, it also offers a quantitative perspective since it attempts to analyze the measurements conducted on said phenomenon. From the data analyzed it was found that women account for the majority in the accounting filed, an aspect that does not necessarily contrast with job remuneration.

Keywords: Feminilization of the accounting profession, feminization of the accounting profession, accounting profession in Colombia, gender, female accountant.

\section{Feminilização e feminização da profissão Contábil na Colômbia}

Resumo: O presente documento tem como objetivo analisar a feminilização e a feminização da profissão contábil na Colômbia entre o ano 2001 e o ano 2018; entendendo como "feminilização" o incremento da participação das mulheres em uma determinada profissão e por "feminização" as mudanças de significado associadas a aumento quantitativo da participação feminina. A pesquisa tem uma perspectiva inicialmente qualitativa que procura compreender e explicar os processos que tem influído na feminização da profissão contábil na Colômbia, através da análise documental; posteriormente é quantitativa porque procura analisar as medições realizadas sobre tal fenômeno. Sobre os dados analisados, encontrou-se que as mulheres constituem uma maioria no campo contábil, fator que não necessariamente se vê contrastado com a remuneração laboral.

Palavras Chave: Feminilização da profissão contábil, Feminização da profissão contábil, profissão contábil na Colômbia, gênero, mulher contabilista.

\section{La féminilisation et la féminisation de la profession comptable en Colombie}

Résumé: ce document a pour but d'analyser la féminilisation et la féminisation de la profession comptable en Colombie depuis l'année 2001 et jusqu'en 2018. La féminilisation comprise comme la hausse de participation de la part des femmes dans une profession particulière, et la féminisation, comme les changements de sens liés à l'accroisement quantitatif de la participation féminine. Cette recherche a d'abord une perspective qualitative qui essaye de comprendre et d'expliquer les processus ayant une incidence sur la profession comptable en Colombie, en faisant appel à l'analyse des documents. Elle est ensuite quantitative car elle veille à analyser les mesures faites sur ce phénomène. Face aux données analysées, il a été trouvé que les femmes constituent la majorité dans le domaine comptable, ce qui n'est pas forcément contrasté avec leur rémunération du travail.

Mots clés: Féminilisation de la profession comptable, féminisation de la profession comptable, profession comptable en Colombie, genre, femme comptable.

1 El rango de tiempo se definió a partir de la información disponible para la investigación en el Observatorio Laboral del Ministerio de Educación Nacional. 


\title{
Feminilización y Feminización de la Profesión Contable en Colombia
}

\author{
Gloria Milena Valero Zapata, Ruth Alejandra Patiño Jacinto y Hasbleidy Vargas Toledo \\ https://doi.org/10.17533/udea.rc.n76a01
}

Primera versión recibida en julio de 2019 - Versión final aceptada en marzo de 2020

\section{Introducción}

I as diferentes y cada vez más numerosas publicaciones acerca de la mujer Lcomo categoría de estudio han propiciado un incremento en las propuestas y debates en torno a su participación activa en el ámbito académico y profesional, en este caso, en particular el contable; por lo cual, los resultados de esta investigación hacen parte de la cuarta fase de un proceso que inició con la indagación sobre el papel de la mujer en el medio académico contable en Colombia, a través de la revisión de los datos presentados en la base de datos del Departamento Administrativo de Ciencia, Tecnología e Innovación [Colciencias] a corte 2010 (Patiño y Valero, 2012); con posterioridad se realizó una revisión documental de las problemáticas y tendencias ideológicas relacionadas con las mujeres desde los años 70 en Europa, Estados Unidos, Canadá, Latinoamérica, Asia y África, identificando generalidades en las condiciones y autores representativos en el tema (Acosta, Patiño, Valero y Díaz, 2015); los resultados fueron utilizados en la tercera fase, la cual se centró en el análisis de una encuesta realizada a un grupo de investigadoras contables latinoamericanas, frente a sus percepciones sobre los diferentes roles que se ejercen como mujer, profesional e investigadora contable, en el marco de los contextos en los que se desempeñan (Patiño, Valero, Acosta y Díaz, En Prensa).

El presente documento tiene como propósito analizar la feminilización y feminización de la profesión contable en Colombia en el período comprendido entre el 2001 y el 2018, entendiendo que la categoría mujer requiere de investigaciones que promuevan análisis desde perspectivas diferentes pero complementarias, como es el caso de la cualitativa y de la cuantitativa; por lo cual, se requiere dar un paso adelante sobre los datos netamente numéricos 
que evidencian el incremento de la participación femenina en el ejercicio de la profesión contable, de forma que se logre dar cuenta de algunos de los aspectos relacionados con este fenómeno, en tanto la creciente participación de las mujeres en las diferentes profesiones liberales que se ejercen en la actualidad.

La investigación desarrollada partió de un enfoque cualitativo que pretendió comprender y explicar los aspectos que han incidido en la feminización de la profesión contable en Colombia, mediante la revisión documental en bases académicas como Redalyc y Spell; a partir de lo encontrado, se desarrolló el enfoque cuantitativo en el que se procedió a revisar la información contenida en el Observatorio Laboral (s.f.) en procura de medir dicho fenómeno. Los conceptos anteriormente mencionados aún no son muy trabajados en el ámbito contable colombiano, por lo cual el estudio es de carácter exploratorio y descriptivo.

Con base en lo anterior, el documento desarrolla los siguiente apartados: en el primero se presenta la contextualización sobre la educación y el ejercicio profesional en Colombia, con la intensión de comprender los desarrollos académicos y profesionales en el país y otorgar un contexto al trabajo; en la segunda parte se presenta un marco referencial de los conceptos de feminilización y feminización, sección que permite reflexionar sobre su naturaleza y comprensión, a partir de la revisión documental de artículos científicos en Latinoamérica, principalmente en Colombia y Brasil; en el tercer apartado se presenta una descripción de la metodología implementada en el desarrollo de la investigación y en particular en el tratamiento de los datos; apartado en el que se analiza los resultados y se presenta la discusión de cada uno de los conceptos (feminilización y feminización) contemplados en el contexto profesional contable colombiano; para cerrar con el desarrollo de las consideraciones finales.

\section{Educación y ejercicio profesional contable en Colombia}

Los principales antecedentes de la profesión contable en Colombia se remontan al siglo XIX con la adaptación del Código Napoleónico, el cual establecía requerimientos sobre libros de comercio y papeles comerciales; con posterioridad, la Misión Kemmerer de 1923 contribuyó con una serie de reformas al sistema financiero y fiscal (Franco, 1998), de tal forma que le garantizará al país capital extranjero; entre las reformas aprobadas a partir la misión, se resaltan los siguientes proyectos de ley, por su relación con la contabilidad:

Ley 34, sobre formación del Presupuesto Nacional, Ley 36, sobre la administración y recaudo de rentas nacionales, Ley 42 , que reorganizó la contabilidad nacional y creó la Contraloría General, Ley 45, que reglamentaba los bancos privados y establecía la Superintendencia Bancaria, y Ley 46 sobre títulos negociables. (Rojas, 2007, p. 81) 
En 1931 la Ley 58 crea la Superintendencia de Sociedades Anónimas, con el propósito de ejercer control y vigilancia sobre estas entidades; sin embargo, es con la Ley 73 de 1935 donde se establece que toda sociedad anónima requeriría un Revisor Fiscal, que principalmente examinará las operaciones, inventarios, actas, libros, entre otros aspectos (Rengifo, 2014).

No obstante, es través del tiempo y frente a las evidencias normativas, que se fue configurando la profesión contable en el país, propiciando un primer acercamiento de formalización profesional en 1887 por la Universidad Externado de Colombia, que centraba su capacitación en la teneduría de libros, con el propósito de iniciar una orientación formal en los temas contables (Rocha y Martínez, 2016). No obstante, es de resaltar que aun cuando no existían los programas de Contaduría Pública como tal, se venían impartiendo asignaturas relacionadas en otras carreras profesionales de la época; así como en las diferentes escuelas de comercio, entre las que vale la pena resaltar la Escuela Nacional de Minas de la Universidad de Antioquia y la Escuela Nacional de Comercio de Bogotá, espacios académicos que contribuyeron a la formación en temas contables.

Con respecto a la Escuela Nacional de Comercio [ENC] de Bogotá, tras diversas reorganizaciones y justamente por ser la única escuela en Bogotá de nivel superior que podía otorgar el título en comercio superior categoría $\mathrm{A}$, se transforma en 1940 "en el centro de preparación de cuadros directivos de nivel medio para la administración pública o las empresas privadas" (Cubides, 1999, p. 67); con posterioridad y en pro de responder a las necesidades económicas del país, se instituye en la ENC el curso de contadores, el cual tendría el respectivo título de contador juramentado, ampliando la formación comercial superior a una carrera profesional intermedia de tres años; lo anterior, producto del Decreto 126 de 1945 (Cubides, 1999).

Entre 1941 y 1945 se expiden una serie de decretos que por una lado tenían como propósito reformar el plan de estudios de comercio superior en la ENC y por otro, estructurar los diferentes tipos de instituciones de formación comercial y los tiempos de formación en cada uno; diferenciando claramente la formación para el trabajo; finalmente es hasta 1951 con el Decreto 356, que la ENC toma el "carácter de Facultad Nacional de Contaduría y Ciencias Económicas, para todo lo relativo a la organización y funcionamiento del curso de Contador Público Juramentado y de los de especialización para técnicos de comercio", y es con este decreto que se da origen a las primeras facultades de Contaduría Pública, las cuales, en sus inicios se caracterizaron por un perfil centrado en la práctica, más que en la academia (Cubides, 1999).

En la actualidad, se encuentran aproximadamente 360 programas de Contaduría Pública profesional activos en el país, en las modalidades presencial y virtual, de los cuales 32 cuentan con Registro de Alta Calidad (Ministerio de 
Educación Nacional, s.f.). Lo anterior evidencia una importancia significativa por formar contadores con una serie de habilidades y competencias, de tal forma que puedan responder a las necesidades de cada región e implicítamente a los requerimientos de las diferentes empresas (Cardona, 2017).

Existe una línea de investigación en educación contable muy desarrollada en Colombia, propiciada por profesores que se han preocupado por analizar y proponer soluciones frente a las problemáticas del entorno; un buen ejemplo de ello son los estudios desarrollados por Cardona y Zapata (2006), en los que se realiza un desarrollo muy importante desde las perspectivas curriculares, los estándares internacionales de educación centrados en competencias, la interdisciplinariedad y la formación integral (Patiño, Valero y Díaz, 2016), la relación de la docencia y la investigación (Patiño, Melgarejo y Valero, 2018), el papel de la pedagogía en la formación contable (Valero, Acosta y Barrios, 2016), los modelos de educación, entre otros temas, los cuales corresponden a un punto de referencia de los trabajos actuales.

Desde este ámbito las preocupaciones han estado vigentes; se ha analizado, escrito y publicado una cantidad importante de investigaciones y reflexiones, con el fin de aportar socialmente desde la disciplina y la profesión contable, posteriormente con la entrada en vigor de los modelos internacionales de educación, toma especial interés en tema de competencias, desde la perspectiva netamente laboral, centrada en cumplirle a las empresas, desconociendo el contexto y los demás actores sociales.

Sobre la perspectiva de competencias y de las tendencias internacionales en educación, provenientes de los procesos económicos, se viene desarrollando una serie de estudios, muchos de los cuales solo contemplan aspectos de formación que contribuyen al gremio empresarial, dejando de lado el aporte social, el cual constituye el más relevante; por lo cual, se investiga temas relacionados con la formación integral, como persona, de tal forma que se logre una perspectiva más global y contextualizada de la formación profesional contable (Valero, Patiño y Duque, 2013), con ello también tratando aspectos como la ética y la perspectiva crítica, la cual se hace necesaria en los profesionales que pretendan aportar desde el cambio de su entorno.

Dados los procesos de internacionalización contable, básicamente la adopción de normatividad internacional, las discusiones se tornan en lo técnico, dejando de lado los aspectos señalados previamente, los cuales constituyen el objeto de la formación contable, la cual debe propiciar capacidades más allá del requerimiento de normativas locales o internacionales (Rueda, Pinzón y Patiño, 2013).

Dentro de este contexto, se evidenciará en este trabajo que las investigaciones en torno a género y contabilidad son muy escasas en Colombia, incluyendo lo relacionado con la perspectiva educativa, por lo cual dentro de las líneas futuras se propone este tema, por ser básicamente inexplorado en el país; 
falta analizar la educación desde una perspectiva particular de género no desde la igualdad, sino justamente reconociendo la diferencia.

\section{Feminilización y Feminización: una aproximación conceptual}

Históricamente el ejercicio profesional ha estado determinado por una marcada diferenciación sexual, que es producto de constructos biológicos, sociales y culturales que permearon las relaciones laborales, de "poder, jerarquía, influencia y dominio, fundamentadas en las relaciones de género" (Lemos, Barufaldi y Paulo, 2015, p. 65); aspectos que han incidido en la segmentación del trabajo, bajo la premisa de que hay trabajos adecuados para hombres y otros para mujeres; por ende el acceso masivo de las mujeres a una determinada profesión u oficio (feminilización), contribuye a generar las transformaciones progresivas y cualitativas en esos espacios laborales (feminización); lo anterior, sumado a la disparidad de género en los cargos de responsabilidad, que ha propiciado el desarrollo de una brecha o muro de cristal (Scott, 2008), donde frecuentemente se favorece al género masculino en términos económicos y de posición, dejando de lado la experiencia o el grado académico que tengan las mujeres en su momento, producto de la generalización de estereotipos e ideas erróneas sobre el deber ser del papel de la mujer en la sociedad, idea apoyada por Giraldo et al. (2011). Es por ello, que es tan valioso entender el cómo y las razones por las cuáles se presentó el cambio, es decir, la feminización de los atributos y características descriptivas que determinan y particularizan la profesión u oficio, así como sus prácticas (Yannoulas, 2011).

Los conceptos de feminilización y feminización parten de un estudio realizado sobre las relaciones de género en el mundo del trabajo, puntualmente sobre los procesos relacionados con la docencia en América Latina y los aspectos que contribuyeron a su desarrollo; de tal forma, la feminilización se entiende como una postura fundamentalmente cuantitativa, centrada en describir y medir el fenómeno, desde el incremento en la participación de las mujeres en una determinada profesión u ocupación. En tanto la feminización, es percibida como una perspectiva cualitativa, que busca comprender y explicar los procesos que han incidido en el aumento de la participación femenina o, como se indicó anteriormente, en el cambio de los atributos del ejercicio profesional (Yannoulas, 2011). No obstante, es importante mencionar que cuando la participación de las mujeres se incrementa notablemente en una profesión es muy posible que "la remuneración total y el prestigio de esta disminuya" (Lemos, Barufaldi y Paulo, 2015, p. 64); como el caso planteado por Bourdieu, Passeron y Mouffe (1971) en Francia, donde "...los maestros de primero y segundo grado, ven disminuido su 'valor' a medida que se feminiza" (p. 74) dicha profesión. 
En la actualidad las mujeres que se inscriben y se gradúan en ciencias contables en Brasil y en Latinoamérica es cada vez mayor; sin embargo, es importante resaltar que aún continúan enfrentándose a ciertas dificultades para alcanzar altos cargos gerenciales, en tanto, se le da mayor peso al rol doméstico que al de profesional contable (Machado, Bernardes y Abbas, 2015).

Frente a los dos conceptos, anteriormente mencionados, es necesario resaltar que en la literatura científica suelen encontrarse como sinónimos, aun cuando dicha diferenciación contribuye a comprender el fenómeno en su amplitud, de tal forma que los análisis cuantitativos evidencian los procesos de transformación de la composición sexual de las profesiones, mientras que los análisis cualitativos propenden por estudios más complejos, que vinculan variables como el poder, la remuneración, la toma de decisiones, entre otros aspectos en el marco del valor social de una profesión u oficio, en la cual la mujer ha incrementado su participación (Yannoulas, 2011).

\section{Metodología}

La presente investigación es cualitativa, ya que pretende comprender y explicar los procesos que han incidido en la feminización de la profesión contable en Colombia, aspecto que se desarrolla a partir del análisis documental de publicaciones académicas relacionadas con la mujer en el ejercicio profesional contable, analizando los debates que se entretejen alrededor; posteriormente, es cuantitativa porque procura analizar e interrelacionar las mediciones realizadas sobre dicho fenómeno, a través de la información contenida en el Observatorio Laboral (s.f.), base de datos que permitirá contrastar y hacer inferencias estadísticas sobre variables como cantidad de graduados por núcleo básico de conocimiento (Contaduría Pública), zona geográfica, origen de la institución de graduación, ingreso promedio y sector de la economía de las mujeres contadoras públicas en Colombia. Este estudio es de carácter exploratorio, en la medida en que en el contexto nacional no se han desarrollado investigaciones que contrasten las razones del incremento en el número de estudiantes y por ende de egresados en la profesión contable; y, es descriptivo, en tanto se centra en dar cuenta de los cambios generados en el ejercicio profesional contable, producto de la feminilización a partir de las variables de análisis propuestas anteriormente.

La investigación se desarrolló en cuatro fases:

Fase I: Esta fase, es fundamentalmente producto de la revisión bibliográfica y el respectivo análisis documental de la información secundaria obtenida de bases científicas como: Redalyc, Scielo y Spell, las cuales fueron seleccionadas justamente por incluir producción académica propia del contexto latinoamericano, lo cual permitió construir el marco referencial del documento, a partir de los conceptos de feminilización y feminización y de 
cómo se han venido dinamizando en los contextos de las fuentes bibliográficas principalmente en Colombia. La revisión bibliográfica tomó como patrones de búsquedas, las siguientes palabras clave: estudios de género, género, feminilización, feminización, contabilidad, contadores, gender, female accounting, feminizing, feminization, women, accounting, accountant, bussiness, senior position, pay. Con los resultados de la revisión se clasificaron los documentos que por su naturaleza tenían relación directa con el contexto colombiano y/o con algún país de Latinoamérica, encontrando varias publicaciones que tomaban como caso de estudio empresas en Brasil; estos documentos permitieron la construcción y sustento argumentativo del apartado dos y de los resultados.

Fase II: Se procedió a obtener la información estadística contenida en el Observatorio Laboral (s.f.) de la siguiente forma:

a) En primer lugar, se analizó la información consolidada de los graduados del núcleo básico Pregrado de Contaduría Pública, en la modalidad presencial, durante el 2001 al 2018, de acuerdo con el sexo (hombre, mujer).

b) Luego se analizaron los resultados anteriormente descritos teniendo como referente la institución universitaria (oficial o privada) con corte a 2015.

c) Para finalmente contrastarlos con los resultados obtenidos en las siguientes zonas geográficas: Antioquia, Atlántica, Bogotá D.C., Central, Oriental, Orinoquía, Amazonía, Pacífica, San Andrés y Providencia, Valle.

d) Con respecto a la situación laboral se analizaron los salarios con corte a 2015, teniendo en cuenta la experiencia laboral relacionada con la fecha de graduación (2001 - 2007 - 2013), y los siguientes sectores económicos: actividades inmobiliarias de alquiler, administración pública y defensa, seguridad social de afiliación obligatoria, agricultura, ganadería, caza y silvicultura, comercio al por mayor y al por menor; reparación de vehículos automotores, motocicletas, efectos personales y enseres domésticos, construcción, educación, explotación de minas y canteras, hoteles y restaurantes, industrias manufactureras, intermediación financiera, organizaciones y órganos extraterritoriales, otras actividades de servicios comunitarios, sociales y personales, pesca, servicios sociales y de salud, suministros de electricidad, gas y agua, y transporte, almacenamiento y comunicaciones. Es importante resaltar que los análisis se realizan de acuerdo con las actividades económicas y no las áreas de desempeño contable, en tanto, la información que se tomó como referente se encuentra clasificada de esa forma.

Fase III: Análisis y discusión de la información encontrada, contrastando los procesos que han incidido en la feminización, frente a la evidencia empírica producto de la fase II y que se concreta en la feminilización de la profesión contable en Colombia. 
Fase IV: se realizan unas breves anotaciones finales y diferentes temas para continuar investigando sobre contabilidad y género.

\section{Resultados}

\section{V.I. Feminización}

Establecer el momento exacto en que la contaduría pública colombiana se feminizó, podría ser una tarea intensa y tal vez infructuosa, en particular porque ha sido un proceso de transformaciones progresivas, determinado por el acceso masivo de mujeres a estudiar dicha profesión; en este apartado se presentan algunas de las razones que evidencian el cambio.

La inclusión de las mujeres propició unas dinámicas de desigualdad profesional que contribuyen con la división del trabajo; en tanto el trabajo reproductivo sigue siendo responsabilidad puntual de las mujeres (Yannoulas, 2011), aspecto que motiva el estudio de la carrera de contaduría pública, con la premisa de que esta profesión permite el desempeño de forma independiente (León y Naranjo, 2011), sin cumplir horarios y posiblemente desde la casa, para continuar ejerciendo sus labores en el hogar. Así mismo se evidencia el progresivo abandono de la profesión contable por parte de los hombres.

Lemos, Barufaldi y Paulo (2015), identificaron una serie de estereotipos relacionados con la inclusión de la mujer en el ejercicio contable entre los que se destaca: "que las mujeres tienen una mejor preparación para las actividades operacionales del área, por ser más detallistas y dóciles que el hombre” (p. 64).

Aun cuando los resultados evidencian una significativa feminilización de la profesión contable en Colombia, dicha incursión no ha logrado feminizar significativamente los cargos de dirección y jerarquía de la profesión; aspecto que propicia escenarios asociados al denominado "techo de cristal" también una tendencia en otros contextos, a partir del estereotipo de género que plantea que "los compromisos familiares limitan el desempeño eficiente de las mujeres en su trabajo" (Giraldo, Jaramillo, Torres y Gómez, 2011, p. 18); dicho estereotipo, es uno de los múltiples aspectos o factores que terminan incidiendo en la baja participación de las mujeres en puestos directivos del área contable; con base en lo anterior, Monllau y Rodríguez (2012) establecen una categorización por obstáculos de estos factores, los cuales se presenta a continuación en la Tabla 1:

2 Entendiéndose como posibles "barreras invisibles que impiden a las mujeres el acceso" (Charlo y Núñez, s.f., p. 2590). 
Tabla 1: Obstáculos relacionados con la baja participación femenina en los puestos directivos del área contable.

\begin{tabular}{ll}
\hline \multicolumn{1}{c}{ Categoría } & \multicolumn{1}{c}{ Característica } \\
\hline $\begin{array}{l}\text { Obstáculos inherentes al } \\
\text { propio género. }\end{array}$ & $\begin{array}{l}\text { "La mujer elige un tipo de vida centrado en la familia, mientras que el hombre } \\
\text { centra su vida en el trabajo". }\end{array}$ \\
$\begin{array}{l}\text { Obstáculos inherentes a } \\
\text { la organización. }\end{array}$ & $\begin{array}{l}\text { Tiene relación con "las preferencias de los clientes de la compañía, (...) las } \\
\text { políticas de recursos humanos de la compañía en aspectos relacionados con las } \\
\text { políticas de promoción y remuneración". }\end{array}$ \\
$\begin{array}{l}\text { Obstáculos de tipo social, } \\
\text { histórico y cultural. }\end{array}$ & $\begin{array}{l}\text { Se explica por los valores y creencias predominantes en un período de } \\
\text { tiempo y en un contexto en particular. }\end{array}$ \\
\hline
\end{tabular}

Fuente: Elaboración propia a partir de (Monllau y Rodríguez, 2012, pp. 83-84).

Así mismo, se deben sumar los resultados de la investigación de Bernardi (1998), quien indica que las mujeres tienen una tendencia a desertar de la carrera de Contaduría Pública, con el propósito de criar a los hijos hasta que estos asistan a la escuela, situación que propicia una desarticulación importante con el ejercicio profesional, lo que compañado de largas horas de trabajo, viajes, estrés y preocupaciones familiares, entre otros aspectos, termina siendo determinante en que las mujeres que logran llegar a cargos de alta gerencia no permanezcan el suficiente tiempo en los mismos. Al contrario, "se produce una 'nueva división del trabajo' que consiste en que las mujeres se han incorporado al mercado laboral sin que los hombres hayan asumido su parte en el trabajo doméstico y de cuidados de la familia” (Pazos, 2008, citado en Monllau y Rodríguez, 2012, p. 91).

\section{V.II. Feminilización}

En los últimos 30 años los incrementos significativos del número de mujeres en la profesión contable han propiciado un sin número de inquietudes relacionadas con su tratamiento frente a: el número de graduadas, los salarios, la experiencia laboral, el entorno de trabajo, la flexibilidad laboral, la satisfacción laboral, las características personales, etc. (Trapp, Hermanson y Turner, 1989); cuestionamientos que son producto justamente de la feminilización de la profesión contable.

Al respecto en Colombia, durante el período comprendido entre el 2001 y el 2018, se encuentran los siguientes resultados. En primer lugar, se evidencia que el valor promedio de graduados por núcleo básico de conocimiento en Contaduría Pública es de un $69 \%$ para las mujeres y de un $31 \%$ para los hombres. En general se puede apreciar una tendencia creciente en el número de mujeres graduadas de la profesión contable frente al número de hombres, como se observa en la Figura 1. 
Figura 1: Graduados por Núcleo Básico Pregrado Contaduría Pública

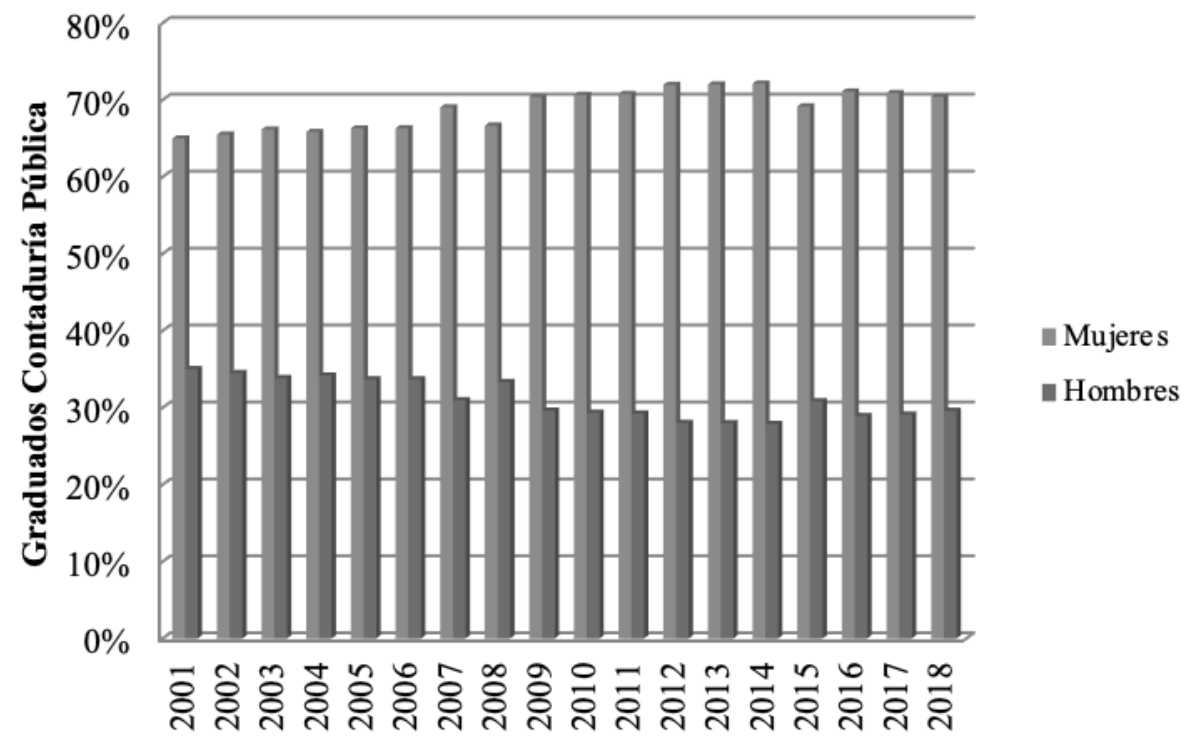

Fuente: Construcción propia a partir de (Observatorio Laboral, s.f.a).

De acuerdo con la naturaleza de la institución de formación, el comportamiento es inversamente proporcional, es decir, en el 2001 se encontró que un $79 \%$ de las graduadas de Contaduría Pública en el país, lo hicieron en instituciones privadas, en tanto que un $21 \%$ en entidades oficiales; en contraste, con los últimos cuatro años de análisis (desde el 2012) donde la relación en promedio es de $45 \%$ del número de graduadas en una institución privada, frente al $55 \%$ en una oficial. Lo anterior implica que las mujeres se han inclinado por una formación en instituciones de carácter público, aspecto que al compararse con los hombres evidencia que el número de graduados en general refleja un notorio descenso en las instituciones privadas, mientras en las públicas se mantiene un promedio del $32 \%$, aspecto que coincide con las consecuencias de la feminilización y de la feminización de la profesión contable en relación a la disminución de profesionales hombres (Lemos, Barufaldi y Paulo, 2015). Ver Figura 2 y 3. 
Figura 2: Graduados Núcleo Básico Pregrado Contaduría Pública por origen institución (mujeres)

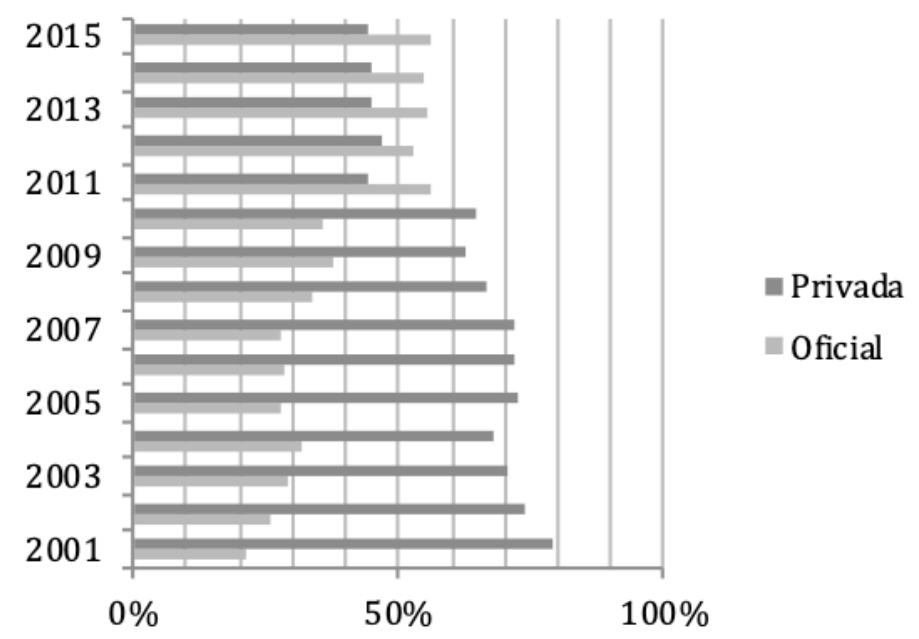

Fuente: Construcción propia a partir de (Observatorio Laboral, s.f.a).

Figura 3: Graduados Núcleo Básico Pregrado Contaduría Pública por origen institución (hombres)

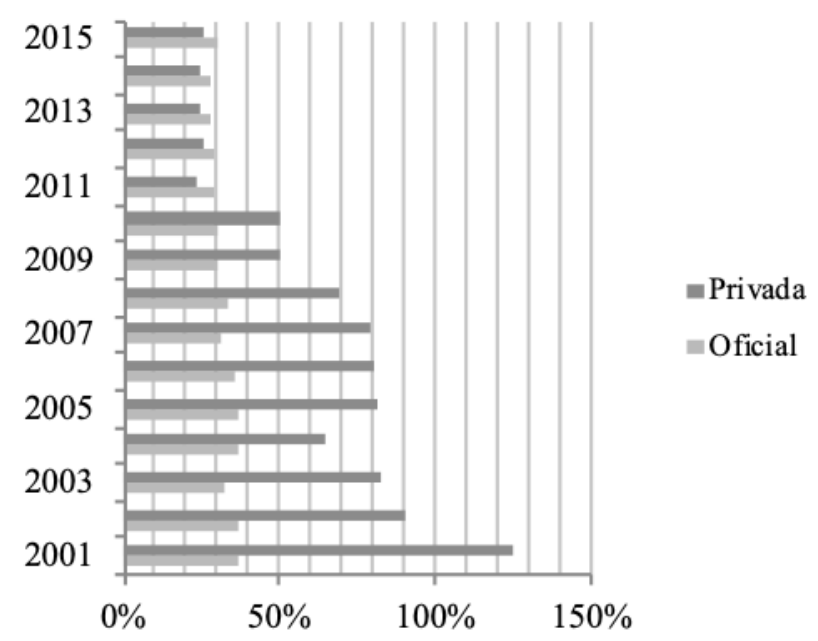

Fuente: Construcción propia a partir de (Observatorio Laboral, s.f.a). 
En cuanto a los resultados por zona geográfica, el incremento del número de graduadas mujeres frente a los hombres es una tendencia constante en el rango de tiempo objeto de análisis, pero que se ha visto duplicado a partir del 2012; situación que podría ser producto del proceso de convergencia a Normas Internacionales de Información Financiera [NIIF], que se consolida partir de la Ley 1314 (2009), la cual contribuye a generar una nueva perspectiva sobre la profesión, con un perfil que requiere nuevos conocimientos y habilidades. Frente a los resultados es importante tener en cuenta que hay zonas en las cuales hay un mayor número de participación de egresados contables, por ser departamentos económicamente claves para el desarrollo del país y de las principales ciudades y departamentos, como es el caso de Bogotá D.C. y Antioquia que reflejan el mayor número de egresados, en tanto, otras zonas agrupan varios departamentos como se evidencia en la Tabla 2.

Tabla 2: Graduados Núcleo Básico Pregrado Contaduría Pública por zona geográfica. ${ }^{3}$

\begin{tabular}{|c|c|c|c|c|c|c|c|c|c|c|}
\hline & & & & & & & Orinoquía & & & \\
\hline & & & Atlántica & & Central & Oriental & Amazonía & Pacífica & & Valle \\
\hline Gep & $\begin{array}{l}\text { Zonas } \\
\text { gráficas / } \\
\text { irtamentos }\end{array}$ & Antioquia & 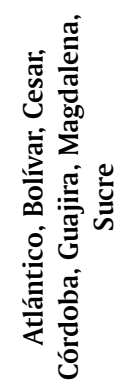 & 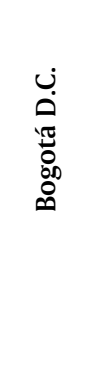 & 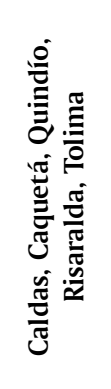 & 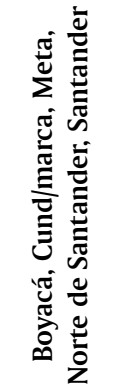 & 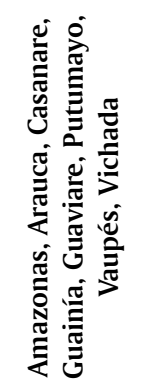 & 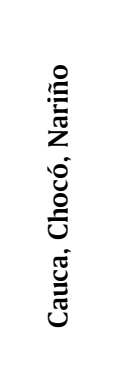 & 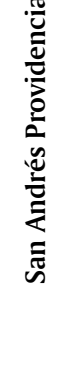 & 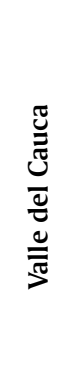 \\
\hline 2001 & Mujeres & 829 & 757 & 2.011 & 301 & 505 & 0 & 100 & 13 & 802 \\
\hline 2001 & Hombres & 256 & 604 & 1.112 & 156 & 209 & 0 & 56 & 10 & 325 \\
\hline 2002 & Mujeres & 968 & 771 & 2.148 & 430 & 597 & 0 & 102 & 13 & 739 \\
\hline 2002 & Hombres & 465 & 604 & 1.072 & 254 & 227 & 0 & 67 & 4 & 339 \\
\hline 2003 & Mujeres & 1.027 & 811 & 1.658 & 817 & 744 & 0 & 66 & 0 & 717 \\
\hline 2003 & Hombres & 405 & 587 & 938 & 424 & 261 & 0 & 66 & 1 & 275 \\
\hline 2004 & Mujeres & 988 & 1.231 & 1.490 & 657 & 591 & 0 & 64 & 0 & 639 \\
\hline 2004 & Hombres & 455 & 826 & 764 & 321 & 239 & 0 & 60 & 0 & 248 \\
\hline 2005 & Mujeres & 783 & 975 & 1.460 & 391 & 546 & 33 & 91 & Nan & 404 \\
\hline 2003 & Hombres & 325 & 703 & 706 & 176 & 244 & 26 & 33 & Nan & 161 \\
\hline & Mujeres & 769 & 654 & 1.879 & 362 & 577 & 0 & 121 & Nan & 454 \\
\hline 2006 & Hombres & 320 & 504 & 949 & 215 & 205 & 0 & 60 & Nan & 197 \\
\hline
\end{tabular}

3 Las zonas geográficas se presentan de acuerdo con la clasificación establecida por el (Observatorio Laboral, s.f.a). 


\begin{tabular}{|c|c|c|c|c|c|c|c|c|c|c|}
\hline & & & & & & & Orinoquía & & & \\
\hline & & & Atlántica & & Central & Oriental & Amazonía & Pacífica & & Valle \\
\hline Gec & $\begin{array}{l}\text { Zonas } \\
\text { gráficas / } \\
\text { rtamentos }\end{array}$ & Antioquia & 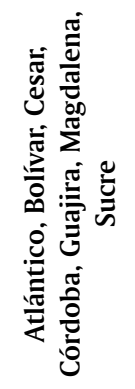 & 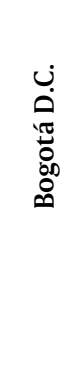 & 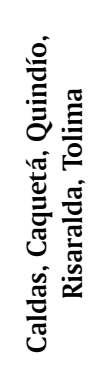 & 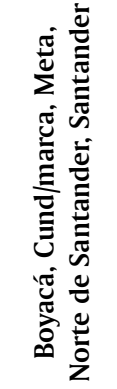 & 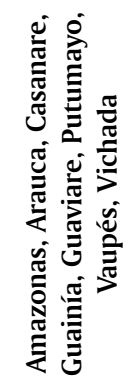 & 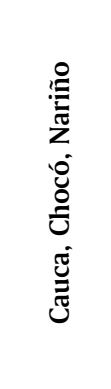 & 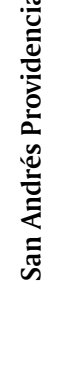 & 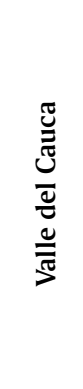 \\
\hline 2007 & Mujeres & 584 & 732 & 1.780 & 551 & 718 & 1 & 213 & 5 & 650 \\
\hline 2007 & Hombres & 229 & 474 & 829 & 260 & 231 & 3 & 118 & 2 & 226 \\
\hline 2008 & Mujeres & 668 & 1.172 & 1.874 & 446 & 794 & 34 & 244 & 13 & 780 \\
\hline 2008 & Hombres & 307 & 905 & 944 & 217 & 292 & 8 & 143 & 3 & 253 \\
\hline 2009 & Mujeres & 980 & 902 & 1.909 & 447 & 591 & 5 & 216 & 0 & 996 \\
\hline 2009 & Hombres & 363 & 555 & 860 & 188 & 196 & 1 & 128 & 0 & 300 \\
\hline 2010 & Mujeres & 1.027 & 895 & 1.867 & 543 & 1.393 & 43 & 287 & 8 & 1.052 \\
\hline 2010 & Hombres & 374 & 616 & 752 & 268 & 447 & 10 & 131 & 3 & 321 \\
\hline 2011 & Mujeres & 1.625 & 1.375 & 3.527 & 888 & 2.054 & 101 & 375 & 17 & 1.572 \\
\hline 2011 & Hombres & 600 & 842 & 1.415 & 428 & 758 & 38 & 200 & 6 & 438 \\
\hline 2012 & Mujeres & 1.738 & 1.477 & 4.045 & 1.313 & 2.363 & 123 & 666 & 15 & 1.747 \\
\hline 2012 & Hombres & 623 & 874 & 1.569 & 571 & 825 & 48 & 335 & 8 & 547 \\
\hline 2013 & Mujeres & 1.701 & 1.717 & 4.955 & 1.396 & 2.502 & 141 & 709 & 20 & 1.789 \\
\hline 2013 & Hombres & 588 & 1.061 & 2.047 & 522 & 908 & 42 & 297 & 6 & 553 \\
\hline 2014 & Mujeres & 1.951 & 1.576 & 4.414 & 1.472 & 2.869 & 262 & 760 & 11 & 1.767 \\
\hline 2014 & Hombres & 658 & 879 & 1.828 & 573 & 1.006 & 94 & 404 & 1 & 537 \\
\hline 2015 & Mujeres & 1.680 & 1.881 & 4.891 & 1.458 & 3.102 & 132 & 726 & 21 & 1.794 \\
\hline 2015 & Hombres & 665 & 1.233 & 2.200 & 683 & 1.099 & 46 & 385 & 9 & 618 \\
\hline
\end{tabular}

Fuente: Construcción propia a partir de (Observatorio Laboral, s.f.a).

Nota: La sigla Nan hace referencia a los períodos de tiempo sobre los que no se obtuvo información.

Conviene precisar que mientras la evidencia empírica demuestra una tendencia constante hacia el incremento en el número de mujeres graduadas de Contaduría Pública en el país, las cifras relacionadas con el salario por sector económico, reflejan una situación muy diferente porque usualmente los salarios están por debajo de los hombres que trabajen en el mismo sector y que puedan tener la misma experiencia laboral; por ende, el análisis no se hace transversal en el tiempo, para ello se efectúan unos cortes que evidencian experiencia 
de 14,8 y 2 años obteniendo los resultados que se describen a continuación ${ }^{4}$, los cuales son coherentes con las investigaciones de Olson y Prieze (citados en Moran, Capozzoli y Ciampa, 1989) que demuestran cómo los hombres y mujeres contables inician sus carreras con un nivel salarial muy similar, pero que en pocos años la mujer puede ganar US $\$ 4.000$ menos por año, frente a los contadores hombres en el contexto estadounidense.

a) La relación de ingresos de mujeres graduadas en el 2001 con corte a 2015 evidencia que el sector más inequitativo es el denominado "Organizaciones y órganos territoriales" con una diferencia salarial de US\$989,58; seguido por el de "Explotación de minas y canteras" con una diferencia salarial de US\$190,05; en tanto que el sector más equitativo es el de "Servicios sociales y de salud" con una diferencia salarial US\$0,98.

b) La relación de ingresos de mujeres graduadas en el 2007 con corte a 2015 evidencia que el sector más inequitativo es el denominado "Explotación de minas y canteras" con una diferencia salarial de US\$1.374,38; seguido por el de "Suministros de electricidad, gas y aguas" con una diferencia salarial de US\$2.972,98; en este rango el sector más equitativo, continúa siendo el de "Servicios sociales y de salud" con una diferencia salarial negativa de US\$70,30; aspecto que evidencia un mejor pago a las mujeres con 8 grados de experiencia, en este sector económico definido por el Observatorio Laboral (s.f.b).

c) La relación de ingresos de mujeres graduadas en el 2013 con corte a 2015 evidencia que el sector más inequitativo es el denominado "Organizaciones y órganos extraterritoriales" con una diferencia salarial de US\$494,45; seguido por el de "Suministros de electricidad, gas y aguas" con una diferencia salarial de US\$247,04; en este rango el sector más equitativo, es el de "Explotación de minas y canteras" con una diferencia salarial negativa de US\$10,09.

Se puede observar que frente a la diferencia salarial, las variables de análisis pueden ser amplias y complejas, situación que seguramente brindará resultados muy particulares que dependerán del sector económico, en la medida en que "el género es significativamente importante en algunos sectores, financiero para hombres y comercio para mujeres" (León y Naranjo, 2011, p. 195); al igual que de otras variables como los años de graduación, la experiencia y los imaginarios profesionales que se tengan; sin embargo, es importante resaltar que las diferencias salariales han disminuido a través del tiempo y de los sectores, situación que evidencia una tendencia positiva de la feminización en la profesión contable colombiana. Por otra parte, Restrepo (2018), respecto al

4 Las unidades monetarias en pesos colombianos se expresan en dólares con una tasa de cambio de $\$ 4,079.96$ COP. 
salario, afirma que la brecha sigue existiendo, pero puede estar explicada por los años de formación en posgrado y la experiencia menor de las mujeres.

De acuerdo con Chamorro (2015) y Chamorro, Patiño y Vásquez (En prensa 2020) la participación femenina en las agremiaciones contables en Colombia sigue siendo bastante baja, mientras que, en temas investigativos, específicamente en publicaciones académicas viene posicionándose, logrando una participación aproximada del $40 \%$.

\section{VI.Conclusiones}

La doble perspectiva replicada en este documento contribuye en alto grado a los diferentes estudios y análisis que se hagan sobre el tema, en la medida en que da cuenta de los valores absolutos y de las características implícitas que han determinado ese fenómeno; ya sea redefiniendo la posición de la mujer, en el caso del presente trabajo en la esfera profesional contable o en el peor de los casos permitiendo el análisis de la mujer en el nuevo escenario de trabajo flexible y mal pago.

En el caso de la profesión contable se evidencia una significativa feminilización del ejercicio profesional, ya que las cifras demuestran de forma evidente el aumento de mujeres contadoras en las distintas zonas en Colombia, lo cual puede explicarse por la posibilidad de trabajo independiente que es compatible con la labor en el hogar; esto es positivo en la medida que la mujer accede al mercado contable profesional, sin embargo, puede ser un limitante para el acceso a cargos directivos y de la misma forma se evidencia que los hijos hacen que las mujeres deserten de sus estudios en Contaduría Pública.

La mayoría de los cargos de alto nivel o de dirección siguen estando en manos de hombres y la remuneración en general se encuentra por debajo de la recibida por contadores con la misma experiencia y en el mismo sector de la economía, inclusive se establece que iniciando su experiencia profesional los salarios de hombres y mujeres son equilibrados y con el pasar del tiempo se van distanciando haciendo que las mujeres contadoras ganen menos que los hombres.

El trabajo evidencia que Colombia se encuentra en un estado de alta feminilización contable, mientras el estado de feminización aún es incipiente, ya que las condiciones siguen siendo inequitativas actualmente. Las causas de ello pueden ser variadas, lo que lleva a la necesidad de seguir estudiando el tema, por ejemplo, en el techo de cristal, las barreras no solo son del medio externo, las mujeres en ocasiones se imponen límites por sus condiciones o intereses. Se evidencia que permanece el techo de cristal en Colombia, ya que no es importante el acceso a cargos de responsabilidad, inclusive considerando que se presentan estereotipos donde frecuentemente se restringe a las mujeres para ocupar cargos de coordinación de equipos de trabajo. 
Es relevante, y como un acumulado de tópicos que se deben seguir estudiando, se plantea la importancia de hacer análisis detallados por área de desempeño contable frente a temas salariales, de ubicación geográfica, así como de tiempo de graduación; es decir, implícitamente de experiencia laboral, de forma que los resultados se puedan comparar con los otros niveles de formación académica como lo son la formación técnica profesional y tecnológica, de la mano de la cantidad de graduados. Con base en lo anterior, se requiere efectuar estudios de caso que den cuenta de los aspectos que propician diferencias salariales de acuerdo con el sexo.

Así mismo sería valioso indagar a profesionales hombres y mujeres de diferentes sectores y períodos de graduación, sobre las particularidades del ejercicio profesional contable femenino; es decir, cuestionar acerca de los atributos y características que la mujer transfiere a la profesión y que por ende la hacen valiosa.

Finalmente, es prioritario desarrollar la línea de estudios de género contable en Colombia, ya que se encuentra en estado inicial; esto, tomando en cuenta que los desarrollos se refieren a tópicos de mujeres y hombres, los estudios aún no contemplan los demás géneros, lo cual es importante en el avance en equidad tomando en cuenta la diversidad. Así mismo, frente a los resultados obtenidos, es imperativo revisar los modelos de enseñanza-aprendizaje propios de la academia contable, donde el contexto es complejo y número de programas muy amplio, de tal forma que se pueda evaluar si dichos procesos están propiciando una especie de "sexismo contable", que contribuye a que las futuras profesionales acepten posiciones de subordinación en la sociedad; entendiendo que mediante la evaluación y diagnóstico se podrá trabajar en modelos que reivindiquen a las mujeres y en general a todo aquel que no encaje en las nociones preestablecidas de hombre y mujer o lo que se conoce como el orden heteronormativo.

En resumen, lentamente se abre camino para distintas formas y nuevos temas de investigación con respecto a género y contabilidad en Colombia, lo cual propondrá soluciones frente a problemáticas y evidenciará nuevas preguntas por responder y discutir.

\section{Referencias bibliográficas}

Acosta, M., Patiño , R., Valero, G. y Díaz, M. (2015). Las problemáticas de género: una introducción para su aplicación en estudios de contabilidad. Revista Activos, 13 (25), 33-72.

Bernardi, R. (1998). The implications of lifestyle preference on a Public Accounting Career: An exploratory study. Critical Perspectives on Accounting (9), 335-351.

Bourdieu, P., Passeron, J. y Mouffe, C. (1971). La independencia, fuente de dependencia del sistema universitario. Ideas y Valores, 20 (38-39), 69-104. 
Cardona, J. y Zapata, M. (2006). Educación contable: Antecedente, actualidad y prospectiva. Medellín: Universidad de Antioquía.

Cardona, J. (2017). Una breve historia de la educación contable en Colombia. Revista Activos, 15(28), 71-81.

Cubides, H. (1999). Evolución de la capacitación y formación de los contadores públicos. Elementos para su interpretación. En H. Cubides, E. Gracia, M. Machado, F. Visbal, \& A. Maldonado, Historia de la Contaduría Pública en Colombia Siglo XX (pp. 35-211). Bogotá: Fundación Universidad Central.

Chamorro, C. (Septiembre de 2015). La participación de la mujer en el desarrollo de la Contaduría Pública. Obtenido de https:/www.researchgate.net/publication/286381703_LA_ PARTICIPACION_DE_LA_MUJER_EN_EL_DESARROLLO_DE_LA_CONTADURIA_ PUBLICA_EN_COLOMBIA

Chamorro, C., Patiño, R. y Vásquez, L. (En prensa 2020). Women accountants in academic and research spaces in Colombia. Advances in Public Interest Accounting.

Charlo, M. y Núñez, M. (s.f.). Introducción de la perspectiva de género en la investigación empírica en contabilidad desde un marco teórico sociológico-institucional. Conocimiento, innovación y emprendedores: Camino al futuro, 2590-2603.

Franco, R. (1998). Contabilidad Integral. Teoría y Normalización (3a edición ed.). Pereira, Colombia: Investigar Editores.

Giraldo, D., Jaramillo, E., Torres, Y. y Gómez, L. (2011). Influencia de los estereotipos de género en el área contable en las grandes empresas de Medellín. Contaduría Universidad de Antioquia (58-59), 197-231.

International Federation of Accountants [IFAC]. (2017, January). Handbook of International Education Pronouncements. Recuperado el 15 de Marzo de 2017, de https://www.ifac. org/publications-resources/2017-handbook-international-education-pronouncements

Lemos, l., Barufaldi, R. y Paulo, N. (2015). La feminización del Área Contable: Un Estudio Cualitativo Básico. Revista de la Academia Brasileña de Ciencias em Contabilidad [REPeC], 9 (1), 64-84.

León, E. y Naranjo, Y. (2011). Profesionales de contaduría pública de Bogotá: trayectoria y prácticas. Tendencias \& Retos, (16), 181-202.

Ley 1314. (13 de Julio de 2009). Por la cual se regulan los Principios y Normas de Contabilidad e Información Financiera y de Aseguramiento de Información aceptados en Colombia. Recuperado el 14 de Enero de 2017, de http:/www.secretariasenado.gov.co/senado/ basedoc/ley/2009/ley_1314_2009.html

Machado, F., Bernades, S. y Abbas, K. (2015). Mulheres no topo: As contadoras paranaenses estao rompendo o glass ceiling? Advance in Scientific and Applied Accounting, 8 (2), 244270.

Ministerio de Educación Nacional. (26 de Enero de 1945). Decreto 126. Por el cual se establecen nuevos cursos en la Escuela Nacional de Comercio. Recuperado el 23 de Marzo de 2020, de Sistema Único de Información Normativa: http://www.suin-juriscol.gov. co/viewDocument.asp?id $=1028110$

Ministro de Hacienda y Crédito Público, encargado del Ministerio de Educación Nacional. (15 de Febrero de 1951). Decreto 356. Por el cual se reforma la Enseñanza Comercial 
Valero, G., Patiño, R., y Vargas, H. Feminilización y Feminización de la Profesión Contable en Colombia

para todo el territorio de la República. Recuperado el 23 de Marzo de 2019, del Sistema Único de Información Normativa: http://www.suin-juriscol.gov.co/viewDocument. asp? ruta $=$ Decretos $/ 1080627$

Ministerior de Educación Nacional. (s.f.). Sistema Nacional de Información de la Educación Superior. Recuperado el 09 de Julio de 2019, de Módulo Consultas: https://snies. mineducacion.gov.co/consultasnies/programa\#

Monllau, T. y Rodríguez, N. (2012). La feminización de las profesiones. Sistema, (228), 8196.

Moran, C., Capozzoli, L. y Ciampa, A. (1989). A Synthesis of Research Studies Regarding the Upward Mobility of Women in Public Accounting. Accounting Horizons, 3(1), 63-70.

Observatorio Laboral. (s.f.a). Perfil de graduados/Graduados por Núcleo Básico de Conocimiento. Recuperado el 18 de Febrero de 2017, de Observatorio Laboral para la Educación: http: http://bi.mineducacion.gov.co:8080/o3web/viewdesktop.jsp?cmnd=open\&sou rce $=$ Perfil + Graduados $\% 2$ FGraduados + por + N\%FAcleo + B $\%$ E1 sico + de + Conocimien to

Observatorio Laboral. (s.f.b). Situación laboral/vinculación - Ingreso y Tasa de Cotización por Nivel de Formación. Recuperado el 27 de Enero de 2020, de Observatorio Laboral para la Educación: http://bi.mineducacion.gov.co:8380/eportal/web/men-observatoriolaboral/sexo

Patiño, R. A. y Valero, G. M. (2012). En clave femenina: La investigación contable Colombiana. Recuperado el 13 de Septiembre de 2014, de Memorias de la XIII Asamblea General de la Asociación Latinoamericana de Facultades y Escuelas de Contaduría y Administración [ALAFEC]: http://132.248.164.227/alafec/docs/asambleas/xiii/ponencias/educacion/ pdf/E_01.pdf

Patiño, R. A., Valero, G. M., Acosta, M. G. y Díaz, M. A. (2021En Prensa). Mujer: experiencias de investigadoras contables en Latinoamérica. Medellin: Fondo Editorial CEIPA, Business School.

Patiño, R. A., Valero, G. M. y Díaz, M. A. (2016). Interdisciplinariedad contable en el contexto profesional actual en las universidades colombianas. Revista ECA Sinergía. Facultad de Ciencias Administrativas y Económicas, 8(1), 63-73.

Patiño, R. A., Melgarejo, Z. A. y Valero, G. M. (2018). Percepción de los egresados sobre la investigación formativa. Revista Activos, 16(30), 101-125.

Rengifo, S. (2014). La revisoría fiscal en Colombia. Recuperado el 29 de Abril de 2019, de Repositorio Universidad Militar Nueva Granada: https://repository.unimilitar.edu.co/ bitstream/handle/10654/13056/La\%20revisor\%EDa\%20fiscal\%20en\%20Colombia.pdf;js essionid $=739$ C78EC00E64F7778448234A0202FFA? sequence $=1$

Restrepo, K. (2018). Diferencias salariales por género en la profesión contable en Colombia (2008-2012). Tesis. Bogotá: Universidad de la Salle.

Rocha, M. y Martínez, I. (2016). Historia de la educación contable en Colombia: la aparición de las primeras escuelas y facultades de Contabilidad. Revista Activos, 14(26), 101-122.

Rueda, G., Pinzón, J. y Patiño, R. A. (2013). Los currículos de los programas académicos de contaduría pública, tras la enseñanza de lo internacional y la globalización en 
la contabilidad: globalización en la contabilidad: necesidades de ajuste más allá de respuestas técnicas. Cuadernos de Contabilidad, 14(35), 639-667.

Rojas, H. (2007). Made in the world is better: las misiones económicas en Colombia y nuestro descreimiento ancestral. Revista Facultad de Ciencias Económicas: Investigación y Reflexión, XV (1), 77-90.

Scott, J. (2008). Género e Historia (1a edición ed.). (I. De Consol, Trad.) México, México: Fondo de Cultura Económica, Universidad Autónoma de la Ciudad de México.

Trapp, M., Hermanson, R. y Turner, D. (1989). Current Perceptions of Issues Related to Women Employed in Public Accounting. Accounting Horizons, 3 (1), 71-85.

Yannoulas, S. (2011). Feminização ou Feminilização? Apontamentos em torno de uma categoria. Temporalis (22), 271-292.

Valero, G. M., Patiño, R. A. y Duque, O. Y. (2013). Competencias para el programa de Contaduría Pública: una aproximación conceptual. Contaduría Universidad de Antioquía. (62), 11-36.

Valero, G. M., Acosta, M. G. y Barrios, S. M. (2016). Caracterización de las didácticas aplicadas en áreas disciplinares en la formación profesional contable en Colombia 2000-2015. CAPIC REVIEW, 14(2), 71082. 